\title{
ADNc RELACIONADOS CON LA MADURACIÓN DEL FRUTO DE GUAYABA (Psidium guajava L.). CARACTERIZACIÓN Y ANÁLISIS DE EXPRESIÓN
}

\author{
RIPENING-RELATED cDNAs IN GUAVA FRUIT (Psidium guajava L.). CHARACTERIZATION \\ AND EXPRESSION ANALYSIS
}

\author{
Alberto I. Reyes Silva ${ }^{1}$, Héctor G. Núñez Palenius ${ }^{2}$, Gustavo Hernández Guzmán², Ángel G. Alpuche Solís ${ }^{3}$, \\ Cristina Garcidueñas Piña ${ }^{1}$ y José F. Morales Domínguez ${ }^{1 \star}$
}

\begin{abstract}
${ }^{1}$ Departamento de Química, Centro de Ciencias Básicas, Universidad Autónoma de Aguascalientes. Avenida Universidad 940, Frac. Ciudad universitaria. 20131, Aguascalientes, Ags. ${ }^{2}$ División Ciencias de la Vida, Campus Irapuato-Salamanca, Universidad de Guanajuato. Ex-Hacienda el Copal, Km 9 Carr. Irapuato-Silao. 36500, Irapuato, Gto. ${ }^{3}$ División de Biología Molecular, Instituto Potosino de Investigación Científica y Tecnología, A.C. Camino a la Presa de San José 2055 , Lomas 4ta. Secc. 78216, San Luis Potosí.
\end{abstract}

*Autor para correspondencia (jfmoral@correo.uaa.mx)

\section{RESUMEN}

En este estudio se presentan los análisis bioinformáticos y de la expresión de cuatro clonas de ADNc que codifican para una poligalacturonasa (PG), para ácido 1-aminociclopropano-1-carboxílico oxidasa (ACCo), y para dos $\alpha$-expansinas ( $\alpha$-Exp) en guayaba (Psidium guajava L.). Mediante RT-PCR se obtuvo un fragmento parcial de 301 pb (PgPG1) correspondiente a una PG que se expresa a partir de fruto maduro, uno de $320 \mathrm{pb}$ (PgACO1) para una ACCo de fruto sobremaduro, y dos para a-expansinas: uno de $466 \mathrm{pb}$ (PgEXP2) de fruto sobremaduro y otro de $362 \mathrm{pb}(P g E X P 3)$ de pedúnculo. El análisis bioinformático de los ADNc mostró que codifican para proteínas putativas con una alta homología con proteínas relacionadas. La secuencia de aminoácidos de la proteína parcial PgPG1 contiene regiones características y conservadas de las PGs en plantas superiores y está relacionada con la maduración de frutos; PgACO1 mostró características presentes en todas las ACCo y está relacionada con la maduración; PgEXP2 y PgEXP3 contienen parte de los dos dominios presentes de las expansinas, y están agrupadas filogenéticamente con las $\alpha$-expansinas. Los estudios de expresión mediante Dot Blot mostraron que el gen PgPG1 fue visible en todos los estadios de maduración del fruto, con mayor intensidad durante el estadio maduro; el gen PgACO1 fue visible en los cinco estadios de maduración del fruto y presentó su expresión más alta durante el estadio de transición, cuando comienza el cambio de color verde al amarillo (estos dos genes muestran comportamientos similares a los reportados en frutos climatéricos); en PgEXP2 la expresión génica se detectó en todos los tejidos, con un incremento a partir del estadio verde 2 al sobremaduro, similar al comportamiento reportado en frutos no climatéricos; para $\mathrm{PgEXP3}$ la expresión fue visible en cuatro estadios de maduración del fruto y en pedúnculo, con mayor intensidad en el estadio maduro que en todos los demás.

Palabras clave: Psidium guajava, reblandecimiento del fruto, ACC oxidasa, expansinas, poligaracturonasas.

\section{SUMMARY}

Bioinformatics analysis and gene expression studies of four clones of guava cDNA encoding for a polygalacturonase (PG), an acid 1-aminocyclopropane-1-carboxylate oxidase (ACCo), and two $\alpha$-expansins ( $\alpha$-Exp) in guava (Psidium guajava $\mathrm{L}$.), is presented here. Using RT-PCR, a partial cDNA fragment of 301 bp (PgPG1) was associated to a PG from mature fruit, one of $320 \mathrm{bp}$ (PgACO1) for an ACCo in overriped fruit, and two fragments for a-expansins: a $466 \mathrm{bp}$ (PgEXP2) of overripe fruit and a $362 \mathrm{bp}$ of peduncle (PgEXP3). cDNA analysis showed that these fragments encode putative proteins with high homology with related proteins. The aminoacid sequence of PgPG1 contains particular features and conserved regions of the PGs in higher plants and is related to fruit ripening; PgACO1 showed features present in all the ACCo and is related to fruit maturation; PgEXP2 and
PgEXP3 partially contain two domains present in expansins; and they are phylogenetically grouped with $\alpha$-expansins. Dot Blot expression studies showed that gene PgPG1 was visible in all stages of fruit ripening, with higher intensity during mature stage. Gene PgACO1 was visible in the five stages of fruit ripening, and was highest during the transition stage (these two genes displayed behaviors similar to those reported in climacteric fruits); PgEXP2 gene expression was detected in all tissues, with an increase from the Green Stage 2 to Overripe Stage 1 , as it has been reported in non-climacteric fruits; for PgEXP3 the expression was visible in four stages of fruit ripening and at peduncle, with highest intensity at the mature stage.

Index words: Psidium guajava, fruit softening, ACC oxidase, expansins, polygaracturonases.

\section{INTRODUCCIÓN}

El proceso de maduración de los frutos es una serie de eventos genéticamente programados, caracterizados por procesos bioquímicos y fisiológicos que alteran su firmeza, color, sabor y textura (Nishiyama et al., 2007). El etileno es la principal molécula involucrada en estos procesos porque coordina numerosas vías metabólicas esenciales en la maduración de los frutos. Su presencia provoca un incremento en los niveles de enzimas hidrolíticas que reblandecen los tejidos, produce hidrólisis de productos almacenados, incrementa la velocidad de respiración y modifica la pigmentación.

Durante la biosíntesis de etileno se lleva a cabo la conversión de S-adenosil metionina (SAM) a 1-aminociclopropano-1-carboxilato (ACC), reacción bioquímica catalizada por la enzima 1-aminociclopropano-1-carboxilato sintasa (ACCs) y la posterior conversión de ACC a etileno catalizada por la ACCo. Por tanto, la modulación de estas enzimas podría cambiar la cantidad de etileno producido y con ello el proceso de maduración (De la Cruz et al., 2010). En este tema se han publicado varios estudios enfocados a dilucidar la participación de genes de ACCo en la producción de etileno durante la maduración de los frutos, como en guayaba enana (Psidium guajava L.) (Agüero et al., 2003) 
y en papaya (Carica papaya L.) (López-Gómez et al., 2004).

El ablandamiento del fruto durante la maduración es un proceso complejo que resulta de numerosas modificaciones en la arquitectura de la pared celular. Durante este proceso, la degradación de pectinas y hemicelulosas contribuye a la pérdida de integridad de la pared celular (Sharova, 2007), degradación que es catalizada por hidrolasas de la pared celular relacionadas con la maduración en frutos. Entre las hidrolasas más estudiadas están las poligalacturonasas (PGs) (Hadfield y Bennett, 1998), que muestran dramáticos incrementos en su actividad y en los niveles de RNAm durante la maduración de frutos como pera (Pyrus communis L.) (Hiwasa et al., 2003) y guayaba (Agüero-Chapin et al., 2006), lo que demuestra el papel importante que juegan en la maduración.

Otras proteínas que modifican a la pared celular durante este proceso son las expansinas (Sharova, 2007), que están involucradas en la extensión y ablandamiento de la pared celular vegetal, mediante la ruptura de los puentes de hidrógeno que mantienen unidas a las cadenas de celulosa y hemicelulosa, sin producir hidrólisis.

En plantas las expansinas tienen diferentes funciones, entre ellas la maduración del fruto. La proteína tiene un peso molecular entre 25 y $27 \mathrm{kDa}$ y contiene dos dominios, uno hacia el extremo amino con estructura y secuencia similar a la familia de las 45 endoglucanasas, e incluye el motivo conservado HFD; el otro dominio está hacia el extremo carboxilo y se ha postulado como sitio de unión a polisacáridos porque posee varios residuos de triptófano (Sampedro y Cosgrove, 2005). Se han identificado, clonado y secuenciado genes de expansinas relacionados con la maduración en especies como tomate (Solanum lycopersicum L.) (Brummell et al., 1999) y fresa (Fragaria $\times$ ananassa D.) (Harrison et al., 2001).

La guayaba es un típico fruto climatérico que exhibe un incremento en la respiración y en la producción de etileno durante la maduración (Jain et al., 2003), gas que juega un papel importante en la regulación de este proceso y el ablandamiento del fruto, de manera que promueve la expresión de genes relacionados con la maduración del fruto, entre ellos los implicados en la modificación de la pared celular, como las PGs (Hiwasa et al., 2003) y expansinas (Sharova, 2007). Jain et al. (2003) registraron la máxima actividad de PGs y de otras enzimas hidrolasas cuando el fruto de guayaba llegaba a sus estadios de maduro y sobremaduro y comenzaba a ser evidente su ablandamiento, de lo cual postularon que la actividad de las PGs contribuye sustancialmente en el ablandamiento de la guayaba. También las proteínas $\alpha$-expansinas se han asociado con el ablandamiento del fruto, como en fresa (Harrison et al.,
2001), y se ha propuesto que contribuyen al ablandamiento al facilitar el acceso de las hidrolasas a los polímeros de la pared celular (Rose y Bennett, 1999).

En comparación con otros frutos climatéricos como tomate, melón (Cucumis melo L.), papaya y otros más, en guayaba se han realizado escasos estudios y avances en el análisis molecular y genético de su maduración, lo que restringe la aplicación de tecnologías moleculares que podrían ofrecer nuevas soluciones a las exigencias actuales para el cultivo de este fruto.

Por ello, el objetivo del presente trabajo fue realizar estudios a nivel bioinformático y molecular para aislar, identificar y caracterizar cuatro secuencias parciales de ADNc que sugieren correspondencia con los genes de ACCo, PGs y $\alpha$-Expansinas, involucrados en la maduración del fruto de guayaba.

\section{MATERIALES Y MÉTODOS}

\section{Material vegetal}

Frutos de guayaba var. 'Media China' de la selección 126, fueron colectados de un único árbol del banco de germoplasma del Campo Experimental "Los Cañones" del Instituto Nacional de Investigaciones Forestales, Agrícolas y Pecuarias (INIFAP), el cual se localiza en el Estado de Zacatecas, México ( $21^{\circ} 45^{\prime} \mathrm{LN}$; $102^{\circ} 58^{\prime} \mathrm{LO}$; $\left.1500 \mathrm{msnm}\right)$. Los frutos fueron clasificados en cinco estadios de maduración, que se caracterizan por diferencias en tamaño, firmeza y color: 1) Verde 1, V1 (100\% verde y pequeño), 2) Verde 2, V2 (100\% verde y mediano), 3) Transición, T (50\% amarillo y $50 \%$ verde), 4) Maduro, M (100\% amarillo), y 5) Sobremaduro, S (80 \% amarillo y $20 \%$ café). En cada estadio se tomaron muestras de frutos por triplicado, así como de hojas y pedúnculos para los análisis moleculares. Todas las muestras fueron inmediatamente congeladas en nitrógeno líquido y almacenadas a $-80^{\circ} \mathrm{C}$ hasta su uso.

\section{Extracción de ARN y análisis RT-PCR}

Se extrajo ARN total de fruto, hoja y pedúnculo con el protocolo descrito por López-Gómez y Gómez-Lim (1992), más un paso adicional para la precipitación de polisacáridos (Asif et al., 2000). El ARN total obtenido se utilizó para la síntesis de ADNc con el paquete ThermoScript ${ }^{\mathrm{TM}}$ RT-PCR System Kit (Invitrogen), con las indicaciones del fabricante. Los ADNc obtenidos fueron utilizados para amplificar los genes de interés mediante la reacción en cadena

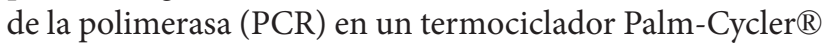
(Corbett Research).

Los dos oligonucleótidos utilizados para PG fueron: F: 
AGYCCIAAYACIGAYGGIRTICA, y R: CARTARTDYTGRTCDATIAYDATIGG; con las siguientes condiciones para la PCR: $94{ }^{\circ} \mathrm{C}$ por $1 \mathrm{~min}, 40{ }^{\circ} \mathrm{C}$ por $2 \min$ y $72{ }^{\circ} \mathrm{C}$ por $3 \mathrm{~min}$, por 35 ciclos (Hiwasa et al., 2003). Para las a-expansinas: F: GSNCAYGCNACNTTYTAYGGNG, y R: YTGCCARTTYTGNCCCCARTT, diseñados de las zonas conservadas de un alineamiento múltiple de varias $\alpha$-expansinas; en este caso las condiciones para la PCR fueron: $94^{\circ} \mathrm{C}$ por $1 \mathrm{~min}, 50^{\circ} \mathrm{C}$ por $1 \mathrm{~min}$ y $72^{\circ} \mathrm{C}$ por $3 \mathrm{~min}$, durante 40 ciclos. Para ACCo: F: GCNTGYSARAANTGGGGNTT, y R: NGGYTCYTTNGCYTGRAAYTT; con las condiciones para la PCR de $94^{\circ} \mathrm{C}$ por $1 \mathrm{~min}, 55^{\circ} \mathrm{C}$ por $2 \mathrm{~min}$ y $72^{\circ} \mathrm{C}$ por $3 \mathrm{~min}$, por 30 ciclos (Nakatsuka et al., 1998).

Los fragmentos amplificados fueron separados por electroforesis en geles de agarosa $1 \%$ mediante la aplicación de $90 \mathrm{~V}$ durante $40 \mathrm{~min}$, y posteriormente teñidos con bromuro de etidio y visualizados bajo luz ultravioleta. Las bandas de interés fueron extraídas del gel de agarosa y purificadas con el método Zymoclean ${ }^{\mathrm{TM}}$ Gel DNA Recovery Kit (ZIMO). Posteriormente, se realizó la secuenciación de cada fragmento con el paquete BigDye ${ }^{\circledR}$ Terminator Cycle Sequencing Kit (Applied Biosystems ${ }^{\circledR}$ ).

\section{Análisis de secuencias}

A cada ADNc se le asignó un nombre correspondiente al gen que presuntamente codifica: $P g P G 1, P g A C O 1, P g E X P 2$ y $P g E X P 3$, y fueron traducidos in silico a secuencias de aminoácidos mediante el uso de la plataforma bioinformática ExPASy (Expert Protein Analysis System) del SIB (Swiss Institute of Bioinformatics) (Artimo et al., 2012) [http:// www.expasy.org]. Estas secuencias se sometieron a una comparación de similitud contra las secuencias del banco de datos, con el programa bioinformático BLAST (Basic Local Alignment Search Tool) del NCBI (Altschul et al., 1990) [http://www.ncbi.nlm.nih.gov]. Con las secuencias de aminoácidos correspondientes a cada ADNc se hizo un alineamiento múltiple con diferentes secuencias de aminoácidos relacionadas, mediante el programa ClustalW2Multiple Sequence Alignment del Instituto Europeo de Bioinformática (EBI) (Larkin et al., 2007) [http://www.ebi. ac.uk]. En cada alineamiento se generó un dendrograma con el método UPGMA basado en el porcentaje de identidad de las secuencias, con el programa Clustal W2 (Larkin et al., 2007). Al final, las cuatro secuencias de ADNc se ingresaron a la base de datos del NCBI.

\section{Análisis de la expresión génica por hibridación Dot-Blot}

Se utilizaron $3 \mu \mathrm{g}$ de ARN total de hoja, pedúnculo y fruto de cinco estadios de maduración, transferidos a una membrana de nilón Hybond ${ }^{\mathrm{TM}}-\mathrm{N}+$ (Amersham) mediante fuerza de vacío. Se utilizó como sonda a los ADNc de PgPG1, PgACO1, PgEXP2 y PgEXP3. Treinta nanogramos de cada ADNc fueron marcados con $\left[\mathrm{P}^{32}\right] \mathrm{dCTP}(3000 \mathrm{Ci}$ nmol $^{-1}$ ), mediante el método Rediprime ${ }^{\mathrm{TM}}$ II Random Prime Labelling System (GE Healthcare). Para cada membrana con su sonda, la hibridación se hizo durante $2 \mathrm{~h}$ a $70^{\circ} \mathrm{C}$ en el amortiguador de hibridación de Amersham Rapid-hyb ${ }^{\mathrm{TM}}$ Buffer (GE Healthcare). Pasado ese tiempo, las membranas se lavaron conforme al protocolo descrito por Sambrook y Russell (2001), y posteriormente se expusieron a una película sensible a rayos-X (Kodak ${ }^{\circledR}$ BioMax ${ }^{\circledR}$ MR film) durante $5 \mathrm{~h}$ a $-70{ }^{\circ} \mathrm{C}$, y finalmente se revelaron.

\section{RESULTADOS Y DISCUSIÓN}

Se obtuvieron cuatro ADNc a partir de ARN total: uno de fruto maduro para una PG ( $P g P G 1)$, dos de fruto sobremaduro, uno para ACCo ( $P g A C O 1)$, y el otro para una expansina ( $P g E X P 2)$; y uno de pedúnculo para otra expansina (PgEXP3). Sus secuencias fueron depositadas en la base de datos del NCBI: PgPG1 (JQ639888), PgACO1 (JQ639887), PgEXP2 (JQ639891) y PgEXP3 (JQ639892).

\section{Estudio bioinformático de los ADNc}

La secuencia parcial de $P g P G 1$ es de 301 pb y codifica para una proteína parcial de 100 aminoácidos (aa). La comparación de esta secuencia en la base de datos del NCBI mostró una identidad por encima de $80 \%$ con otras secuencias de aminoácidos correspondientes a PGs de plantas superiores, relacionadas con la maduración del fruto. El alineamiento múltiple con otras seis secuencias de aminoácidos de PGs específicas de la maduración (Figura 1), mostró resultados similares con respecto a frutos de pera (Hiwasa et al., 2003). El análisis de la secuencia de aminoácidos mostró que la secuencia PgPG1 contiene regiones características y conservadas de las PGs: una rica en glicina (recuadro en Figura 1), que está altamente conservada en muchas PGs de plantas, hongos y bacterias; un residuo de histidina, al que Hadfield et al. (1998) le atribuyen una actividad catalítica; cuatro dominios conservados (regiones en gris, Figura 1); y dos de ocho residuos de cisteínas.

El dendrograma mostró tres grupos (Figura 2): el Grupo A que incluye a las PGs que se expresan en fruto y en zona de dehiscencia; el Grupo B donde PgPG1 está agrupada, que incluye a PGs que se expresan en fruto y zona de abscisión; y el Grupo C que incluye a PGs expresadas en polen y anteras; $y$ una secuencia de PG como grupo externo correspondiente a Aspergillus flavus. PgPG1 contiene una identidad cercana con la PcPG2 de pera que es específica de la maduración del fruto (Hiwasa et al., 2003), resultado que es congruente porque se utilizaron los mismos oligonucleótidos en ambos trabajos. 


\begin{tabular}{|c|c|}
\hline $\mathrm{PC}-\mathrm{PG} 2$ & PNTDG I HVQMS SGVTILDSKISTGDDCVSVGPGTTNLWIENVACGPGHG IS IGSLGKDQQEAGVQNVTVKTVTFI \\
\hline PgPG1 & PNTDXVHIGASTGVTILNSKIGTGDDCVSIGPGSNNLWIENLACGPGHG IS IGSLGKDQQEDGVQNVTVKTVTFI \\
\hline APG2 & PNTDG I HVKLSSGVS I INSH I GTGDDC IS I GPGT SNLW IEG I ACGPGHG I S I GSLGWKQQELGVQNVTVKTVT \\
\hline PG1 & PNTDGI HVERSSNVTILNSNIRTGDDC IS I GPGTSHLWMERLACGPGHG IS IGSLGKWWEEAGVENVTLKTA \\
\hline uayabaPG & ---IGIHVGRSTGVNITGADIRTGDDCVSLGDGSQQVNVESVTCGPGHG IS IGSLGKYHDEQPVVGVTVRNC \\
\hline PG3 & PNTDGIHVTGTQF IVIKNCLIMTGDDC IS IVSGSKNVRAKG I TCGPGHG IS IGSLGAGKSEAEVSNVVVDTA \\
\hline \multirow[t]{2}{*}{$\mathrm{PC}-\mathrm{PG} 1$} & PNTDGIHVTNTQNITISSSVIGTGDDCISIVSGSQRVQATDITCGPGHGISIGSLGEDGSKDHVSGVCVNGA \\
\hline & $: * \star \star \star * \star * \star * \star * \star *$ \\
\hline C-PG2 & GVRIKSWGRPSTGFARS ILFQHIVMTNVQNPIVIDQ-NYCPND-KGCPGQASGVKVSDVTYQGIHGTSAT 34 \\
\hline $9 P G 1$ & GVRIKSWARPSNSFARNILFQ------------------------------------------1 10 \\
\hline APG2 & GVRVKTWARPSNGFVRNILFQHIVMVNVKNPIIIDQ-NYCPNH-ESCPHQGSGIKISDITYQDIHGTSAT 33 \\
\hline PG1 & GVRIKSWGRPSNGFAKNILFKHIVFDNVNNPLIIDQ-NYCPHN-QGCPGQASGVKISNVRYEDIHGTSAT 34 \\
\hline uayabaPG & GIRVKTWPASPGGVATNMHFEDITVKNVSTPILIDQKNYCPSI------------------------ 119 \\
\hline PG3Melon & GVRIKTWQGGK-GYAQNI IFQNIVMDNVTNPIIINQ-NYCDQK-EPCTQQADAVAVSNVMYQNIRGTSAS 402 \\
\hline C-PG1Pera & $\begin{array}{l}\text { GLRIKTWQGGS-GSATNIVFQNVQMNNVTNPIIIDQ-NYCDHKTKDCKQQKSAVQVKNVLYQNIRGTSAS } 414 \\
\star \star \star * \star *\end{array}$ \\
\hline
\end{tabular}

Figura 1. Alineamiento múltiple de la proteína parcial PgPG1 y de seis secuencias de aminoácidos que codifican para proteínas poligalacturonasas, PGs. Pera PC-PG2 [No. Acceso BAC22689], tomate TAPG2 [No. Acceso AAB09575], melón MPG1 [No. Acceso AAC26510], GuayabaPG [No. Acceso AAX12520], melón MPG3 [No. Acceso AAC26512] y pera PC-PG1 [No. Acceso BAC22688]. El alineamiento se obtuvo con el programa Clustal W2 (Larkin et al., 2007). Los guiones indican "huecos" introducidos para maximizar la similitud. Los asteriscos indican secuencias idénticas. En un cuadro se muestra una región rica en glicina altamente conservada en PGs de plantas, hongos y bacterias; y el símbolo • dentro de esta región señala un residuo de histidina encontrado en todas las PGs secuenciadas. En negritas se muestran potenciales sitios de glicosilación $\mathrm{N}-\mathrm{X}-\mathrm{T} / \mathrm{S}$ (Hadfield et al., 1998). En sombreado gris se marcan dominios conservados de PGs (Gorguet et al., 2009). Los dos puntos (:) indican residuos de cisteína altamente conservados entre PGs de especies de plantas superiores y hongos (Redondo-Nevado et al., 2001).
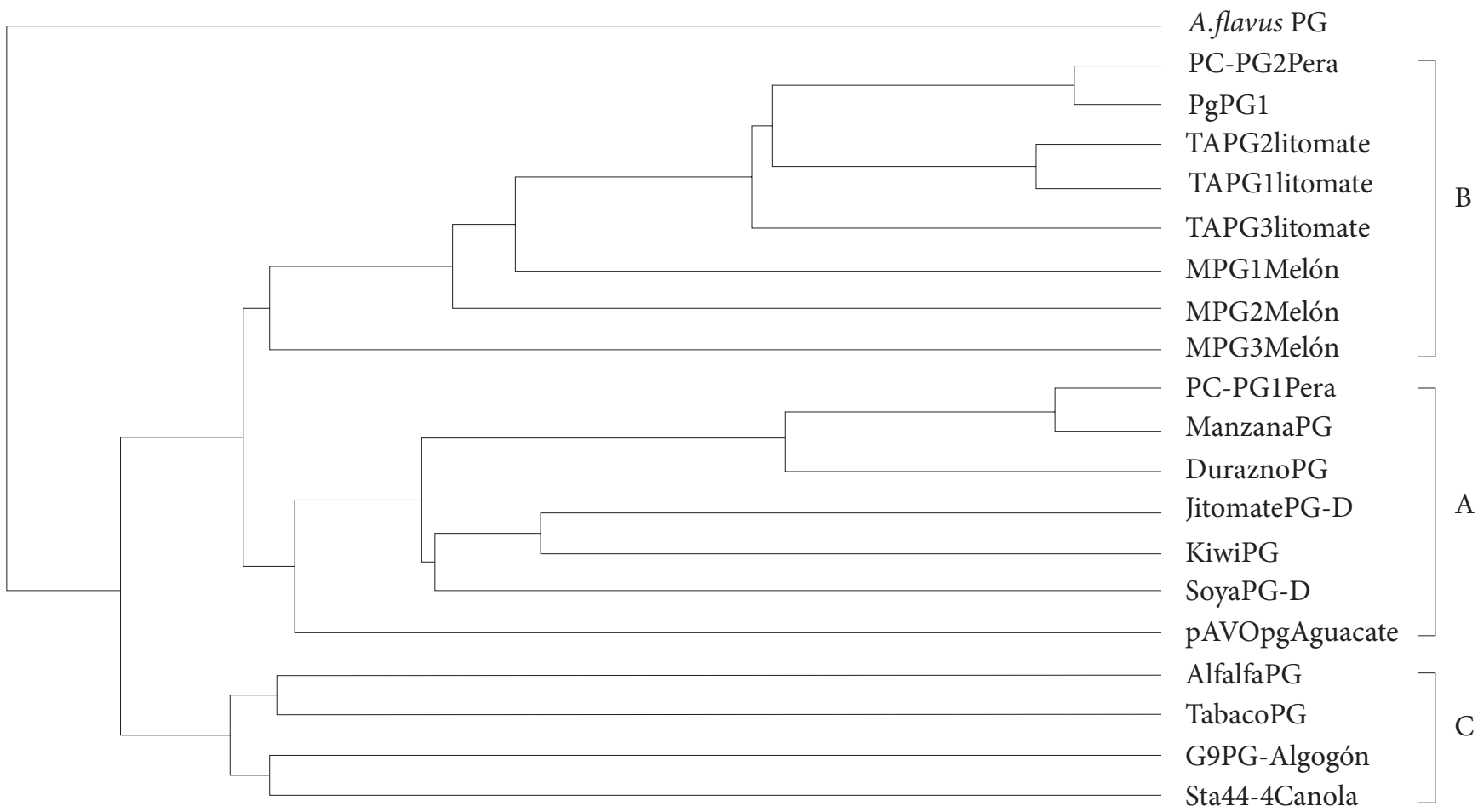

Figura 2. Dendrograma diseñado a partir del porcentaje de identidad entre 19 secuencias de aminoácidos correspondientes a PGs y a la proteína parcial PgPG1, con el programa Clustal W2 (Larkin et al., 2007). Las PGs se agrupan en tres diferentes grupos denominados A, B y C (Hadfield et al., 1998), donde PgPG1 se ubica dentro del grupo B. Se incluye una PG de Aspergillus flavus como grupo externo. 
El hecho de que el dendrograma separe tres grupos, apoya la hipótesis de que PgPG1 tiene una actividad de endopoligalacturonasa, ya que según Hadfield et al. (1998) la separación de PGs implicadas en procesos de abscisión y de maduración del fruto, con respecto a las de polen y anteras, puede reflejar una diferencia funcional de la actividad endo y exo enzimática entre esos dos grupos; esto también es apoyado por los estudios filogenéticos de PGs (Hiwasa et al., 2003; Hadfield et al., 1998). Estas características, junto con la identidad tan estrecha con otras PGs que mostró el dendrograma, permitieron sugerir que el gen $P g P G 1$ codifica para una PG.

La secuenciación parcial de $P g E X P 2$ y $P g E X P 3$ fue de 466 y 362 pb respectivamente, las cuales codifican para dos proteínas parciales de 154 y 120 aa. La comparación con la base de datos mostró una identidad por encima de 80 $\%$ con otras secuencias de aminoácidos correspondientes a expansinas. El alineamiento de las proteínas parciales PgEXP2 y PgEXP3 con cinco secuencias de aminoácidos correspondientes a a-expansinas específicas de la maduración, mostró características similares con los dos dominios presentes en las expansinas (Figura 3); entre éstas están siete de los ocho residuos de cisteínas, el motivo conservado histidina-fenilalanina-aspartato (HFD) en el dominio I (Sampedro y Cosgrove, 2005).
El dominio II se caracteriza por la presencia de cuatro residuos de triptófano (W) conservados que potencialmente forman un dominio de unión a celulosa (Civello et al., 1999) y que están presentes en todas las $\alpha$-expansinas y $\beta$-expansinas, como en las de Zea mays L. (Wu et al., 2001). En las secuencias de este estudio sólo la proteína PgEXP2 mostró dos residuos de $\mathrm{W}$, debido a que la secuencia es parcial y por su reducido tamaño no se logró corroborar la presencia de los demás residuos de $\mathrm{W}$.

De acuerdo con el dendrograma, las proteínas parciales PgEXP2 y PgEXP3 poseen una estrecha identidad con otras a-expansinas, entre éstas la PgEXP1 (núm. acceso JQ639890), que corresponde a una expansina involucrada en la maduración del fruto de guayaba (Figura 4). En el mismo dendrograma se observan otros tres grupos: uno de $\beta$-expansina, otro parecido a $\alpha$-expansina, y el tercero parecido a $\beta$-expansina, más una secuencia parecida a una expansina de Dictyostelium discoideum como grupo externo. Esta agrupación en cuatro diferentes familias concuerda con la superfamilia de expansinas descrita por Choi et al. (2006).

La secuencia parcial del gen PgACO1 es de 320 pb y codifica para una proteína parcial de 106 aa. La comparación con la base de datos mostró una identidad por encima de $80 \%$ con otras enzimas ACCo de plantas superiores, la

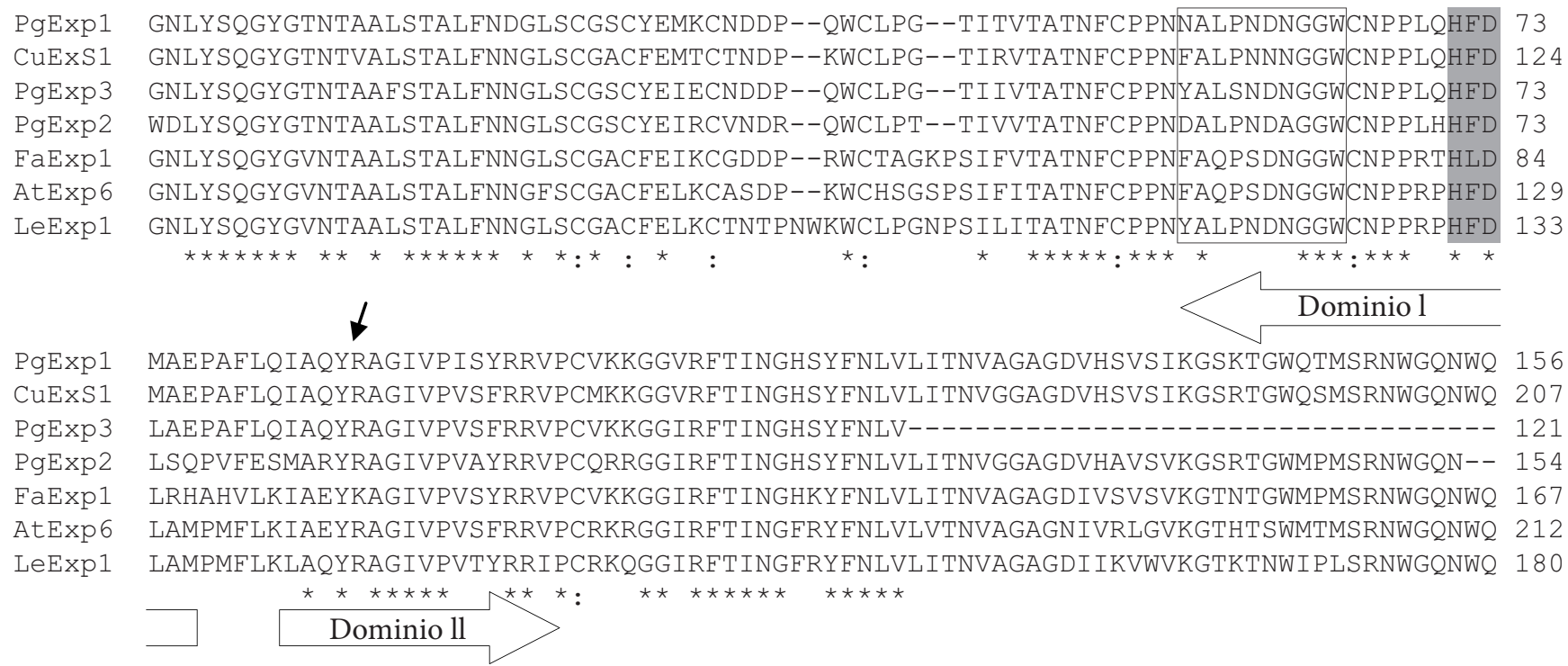

Figura 3. Alineamiento múltiple de las proteínas PgEXP2, PgEXP3 y cinco secuencias de aminoácidos de a-expansinas. Guayaba PgEXP1 [No. Acceso AFH02979], pepino CuExS1 [No. Acceso AAB37746], fresa FaExp1 [No. Acceso AAD44345], Arabidopsis thaliana AtExp6 [No. Acceso NP180461] y tomate LeExp1 [No. Acceso AAC63088]. El alineamiento se obtuvo con el programa Clustal W2 (Larkin et al., 2007). Los guiones indican "huecos" que fueron introducidos para maximizar la similitud. Los asteriscos indican secuencias idénticas. Los dos puntos (:) señalan residuos de cisteína conservados en todas las expansinas (Brummell et al., 1999). El límite entre el dominio I y II está indicado por flechas. En sombreado gris se muestran los residuos (HFD) conservados en el dominio I de algunas $\alpha$-expansinas y $\beta$-expansinas. La flecha negra señala un sitio de deleción, y enmarcado en un cuadro se encuentra una región de inserción, ambos característicos de las a-expansinas (Sampedro y Cosgrove, 2005). 


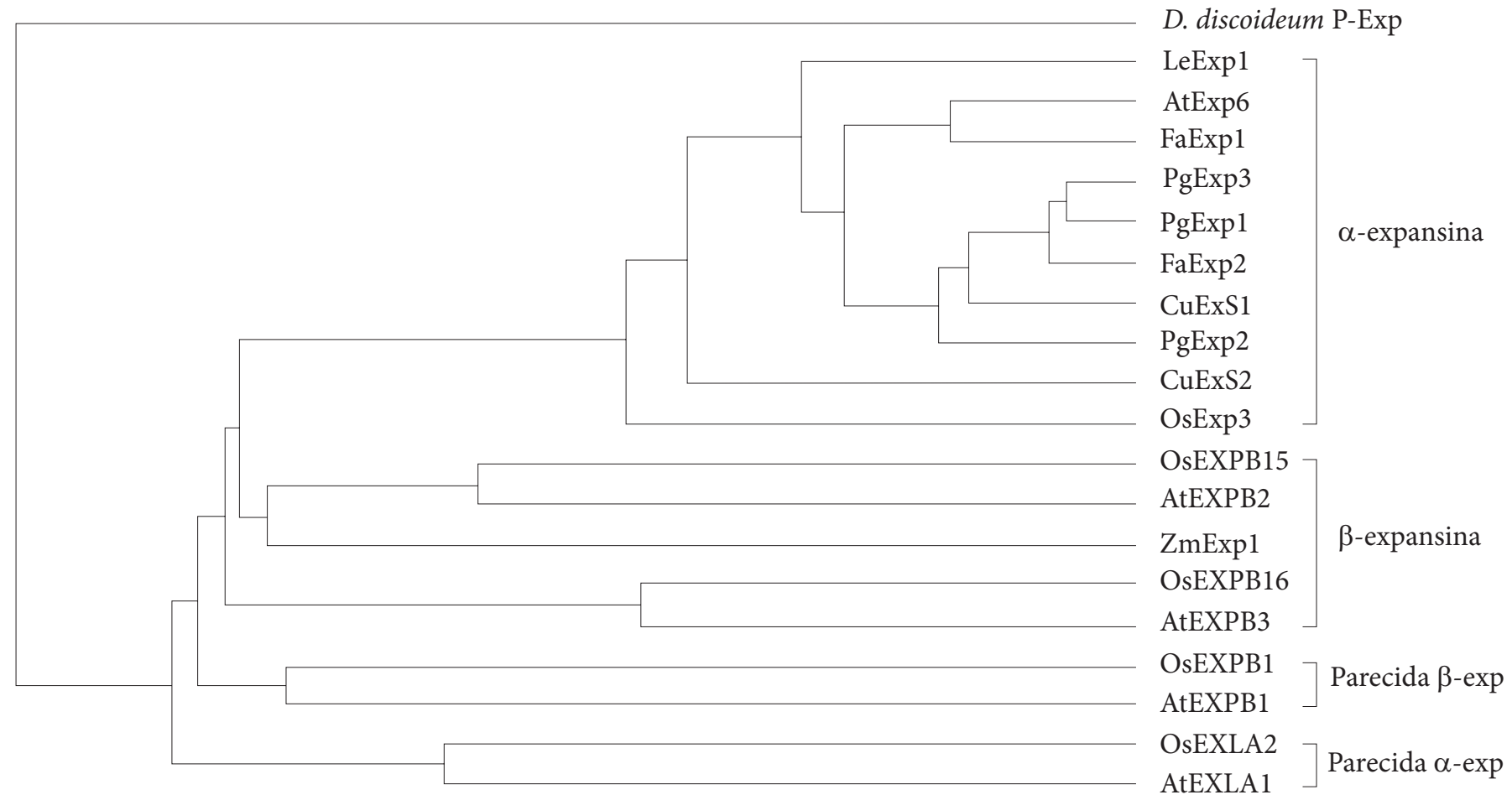

Figura 4. Dendrograma diseñado a partir del porcentaje de identidad entre 17 secuencias de aminoácidos correspondientes a expansinas y a las proteínas parciales PgEXP2, PgEXP3, hecho con el programa Clustal W2 (Larkin et al., 2007). Las secuencias pertenecientes a la superfamilia de expansinas se agrupan en cuatro familias: “ $\alpha$-expansinas, $\beta$-expansinas, parecidas a $\alpha$-expansinas y parecidas a $\beta$-expansinas” (Sampedro y Cosgrove, 2005), donde PgEXP2 y PgEXP3 están incluidos dentro de la familia a-expansina. Como grupo externo se usó una secuencia de una proteína parecida a expansina.

mayoría relacionadas con la maduración del fruto. El alineamiento con otras seis secuencias de aminoácidos correspondientes a ACCo (Figura 5), mostró sólo dos de ocho regiones altamente conservadas entre todas las secuencias de ACCo (Momonoi et al., 2007). Sin embargo, en el dendrograma (Figura 6) se aprecia una identidad estrecha con otras secuencias de ACCo, en particular con una involucrada en la maduración del fruto de la guayaba 'Enana' (Agüero et al., 2003). Este dendrograma detectó cuatro grupos que pertenecen a la super-familia de Fe(II) ascorbato dioxigenasa, en concordancia con estudios de otras ACCo (Tang et al., 1993). El Grupo A, donde PgACO1 está agrupada, incluye secuencias de ACCo que se expresan en la maduración del fruto, así como en respuestas a heridas de hoja y al marchitamiento de pétalos, tallos y hojas; los Grupos B, $\mathrm{C}$ y D incluyen secuencias correspondientes a las proteínas E8, flavonona 3-hidroxilasas y A2, respectivamente. Como grupo externo se incluyó una secuencia correspondiente a ACC deaminasa de Pseudomonas fluorescens.

\section{Análisis de expresión}

La expresión de los diferentes ADNc se analizó en hoja, pedúnculo y cinco estadios de maduración del fruto, como se muestra en la Figura 7. La expresión mediante Dot Blot de PgPG1 fue visible en todos los estadios de maduración del fruto y en pedúnculo, aunque la expresión fue mayor durante el estadio $\mathrm{M}$ al igual que en otros frutos como pera (Hiwasa et al., 2003), lo que sugiere un incremento en la actividad de PGs con la concomitante degradación de polisacáridos de pectina y el reblandecimiento característico del fruto en este estadio del fruto. Además, nuestros resultados concuerdan con los estudios bioquímicos realizados por Jain et al. (2003), quienes observaron cambios en la actividad de las PGs durante la maduración, y una alta correlación entre actividad de la enzima y la pérdida de firmeza en el fruto.

Por ello se puede inferir que la expresión del gen $P g P G 1$ posee una relación con la pérdida de firmeza del fruto, al igual que otros genes reportados en especies como melón (Hadfield et al., 1998). No obstante, este gen también presenta una expresión elevada en el estadio del fruto V2, durante el cual no ocurre reblandecimiento. Es decir, en guayaba no hay una clara relación entre la expresión del gen y el proceso de ablandamiento del fruto; un patrón semejante fue reportado en fruto de fresa por RedondoNevado et al. (2001), quienes propusieron que la actividad 
PgACO1

GuayabaACO

$\mathrm{LE}-\mathrm{ACO} 1$

$\mathrm{LE}-\mathrm{ACO} 3$

PetuniaACO 3

$\mathrm{LE}-\mathrm{ACO} 2$

TulipanACO3

PgACO1

GuayabaACO

$\mathrm{LE}-\mathrm{ACO} 1$

$\mathrm{LE}-\mathrm{ACO} 3$

PetuniaACO3

$\mathrm{LE}-\mathrm{ACO} 2$

TulipanACO3

MDTVERLTKGHYKKCMEQRERELVASKGLEYVQKEVHDLDWESTFHLKHLPESNIFQIPDLDDDYRKVMKEFAVK 74 MDTIERMTKGHYKKCMEQRERELVVSKGLEYVQKEVHDLDWESTFHLKHLPESNISQISDLDDDYRKVMKEFAVK 91 MDTVEKMTKGHYKKCMEQRFKELVASKGLEAVQAEVTDLDWESTFFLRHLPTSNISQVPDLDEEYREVMRDFAKR 120 MDTVEKLTKGHYKKCMEQRFKELVASKGLEAVQAEVTDLDWESTFFLRHLPTSNISQVPDLDEEYREVMRDFAKR 120 MDTVEKLTKGHYKKCMEQRFKELVASKGLEAVQAEVTDLDWESTFFLRHLPVSNISEVPDLDDEYREVMRDFAKR 120 MDTVEKLTKGHYKKCMEQRFKELVAKKGLEGVEVEVTDMDWESTFFLRHLPSSNISQLPDLDDVYREVMRDFRKR 120 LDKVERLTKDHYKKCMEERFREFASKTLQDGSKVDVDNLDWESTFYLRHLPTSNMSEIPDLSDEYRETMKEFALR 120

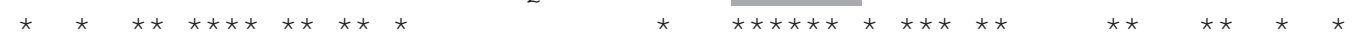

LEKLAEELLDLLCENLGLEKGYLKNAFHGSN------------------------- 105

LEKLAEQLLDLLCENLGLEKGYLKKAFYGSNGPTFGTKVSNYPPCPKPDLIKGLRAHTDA 151 LEKLAEELLDLLCENLGLEKGYLKNAFYGSKGPNFGTKVSNYPPCPKPDLIKGLRAHTDA 180 LEKLAEELLDLLCENLGLEKGYLKNAFYGSKGPNFGTKVSNYPPCPKPDLIKGLRAHTDA 180 LEKLAEELLDLLCENLGLEKGYLKKAFYGSKGPNFGTKVSNYPPCPKPDLIKGLRAHTDA 180 LEKLAEELLDLLCENLGLEKSYLKNTFYGSKGPNFGTKVSNYPPCPKPDLIKGLRAHTDA 180 LEELAEQLLDLLCENLGLEKGYLKKAFSGSKGPTFGTKVSNYPPCPKPELIKGLRAHTDA 180

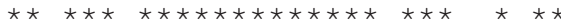

Figura 5. Alineamiento múltiple de la proteína parcial PgACO1 y seis secuencias de aminoácidos que codifican para enzimas de ACCo. GuayabaACO [No. Acceso AAM74522], tomate LE-ACO1, LE-ACO3, LE-ACO2 [No. Acceso PO5116, CAA68538, P24157], petuniaACO3 [No. Acceso AAA33697] y tulipán ACO3 [No. Acceso BAE20197]. El alineamiento se obtuvo con el programa Clustal W2 (Larkin et al., 2007). Los guiones indican "huecos" que fueron introducidos para maximizar la similitud. Los asteriscos indican secuencias idénticas. Las regiones altamente conservadas en ACCo se marcan con sombreado (Momonoi et al., 2007).

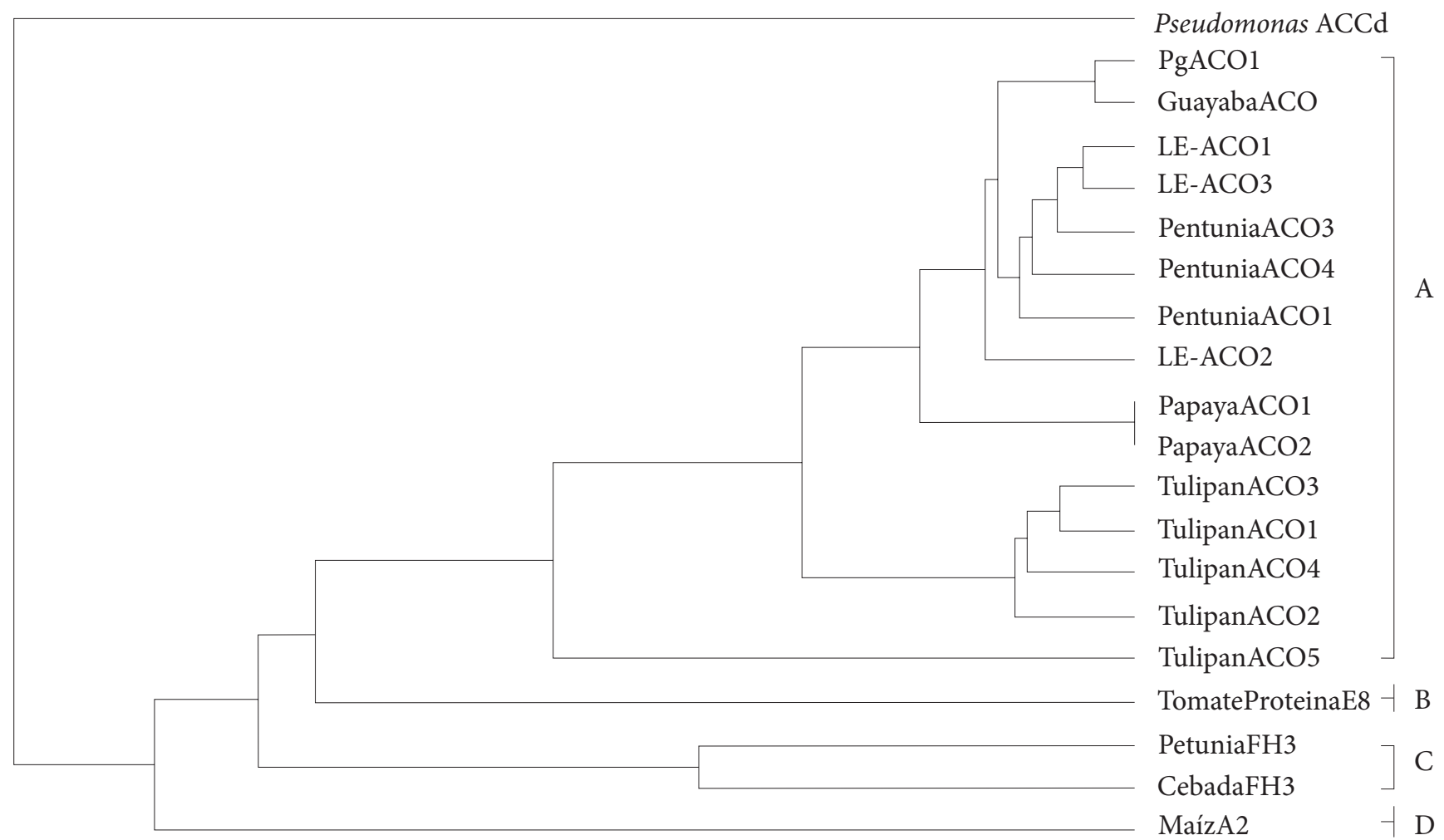

Figura 6. Dendrograma diseñado a partir del porcentaje de identidad entre 18 secuencias de aminoácidos correspondientes a la superfamilia $\mathrm{Fe}(\mathrm{II})$ y ascorbato, y la secuencia putativa PgACO1, hecho con el programa Clustal W2 (Larkin et al., 2007). Las secuencias pertenecientes a la superfamilia $\mathrm{Fe}(\mathrm{II})$ y ascorbato se agrupan en cuatro familias A, B, C y D (Tang et al., 1993), donde PgACO1 se ubica dentro de la familia A correspondiente a ACCo. Como grupo externo se usó una secuencia de aminoácidos correspondiente a una ACC deaminasa de Pseudomonas fluorescens. 


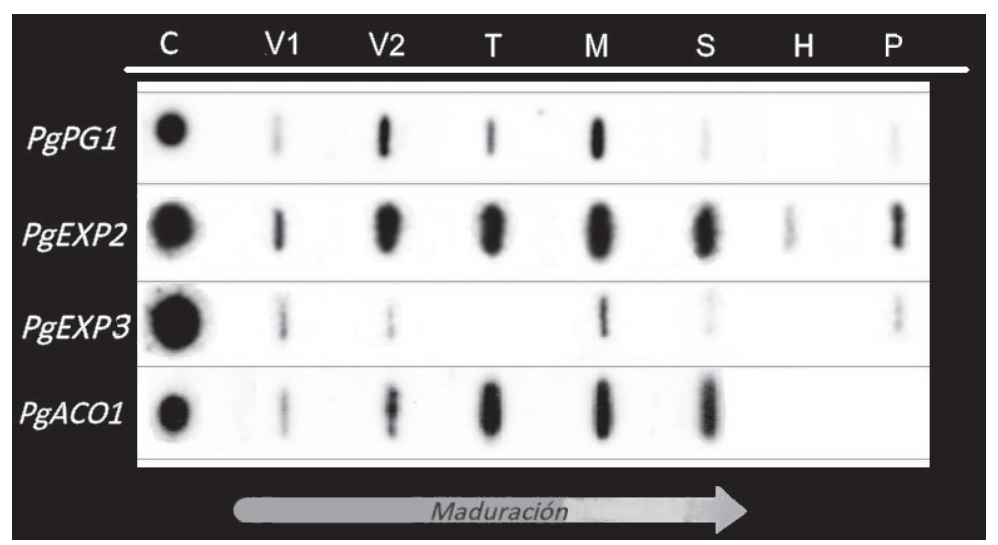

Figura 7. Análisis de expresión por Dot Blot de los genes PgPG1, PgEXP2, PgEXP3 y PgACO1 en hoja, pedúnculo y en fruto de guayaba durante su desarrollo y maduración. Columna $\mathrm{C}$, control positivo; V1, estadio en fruto verde 1; V2, estadio en fruto verde 2; T, estadio en fruto transición; $M$, estadio en fruto maduro; $\mathrm{S}$, estadio en fruto sobremaduro; $\mathrm{H}$, hoja; $\mathrm{P}$, pedúnculo. La flecha indica el progreso de maduración del fruto de guayaba.

de dicha enzima sobre las pectinas genera oligosacarinas biológicamente activas, las cuales participan como señales moleculares potencialmente involucradas en la regulación de algunos procesos metabólicos relacionados con la maduración del fruto.

En el caso del gen PgEXP2 la expresión fue visible en todos los tejidos, con un incremento en fruto desde el estadio V2 hasta el S. Este patrón de expresión coincide con los cambios de expresión bajo-alto-bajo observados en una $\alpha$-expansina del fruto de fresa (Harrison et al., 2001), la cual también está presente en raíz, hoja, estolón y fruto; en este último se expresa durante su desarrollo y maduración.

Para PgEXP3 la expresión fue visible en cuatro estadios de maduración del fruto y en pedúnculo, y fue mayor en el estadio $\mathrm{M}$ que en todos los demás. Este patrón de expresión alto-bajo-alto, también se observó en expansinas de fruto de fresa (Harrison et al., 2001). Por tanto, los genes de estas dos expansinas, $P g E X P 2$ y $P g E X P 3$, participan de manera simultánea en el desarrollo y maduración del fruto de guayaba. Según Brummell et al. (1999), al menos seis genes de $\alpha$-expansinas se expresan durante el desarrollo del fruto en tomate, y tres de ellos también se expresan en hoja y flores, lo que muestra la expresión simultánea de varias expansinas.

La expresión del gen PgACO1 fue visible en los cinco estadios de maduración del fruto, y alcanzó su máximo nivel de expresión durante el estadio $\mathrm{T}$, para luego disminuir durante los estadios M y S. Este resultado sugiere que hay cambios en la actividad enzimática de ACCo y en la concomitante biosíntesis de etileno. Este patrón de expresión también se ha observado en otros frutos climatéricos, como papaya (López-Gómez et al., 2004).

Se espera que la información aquí obtenida contribuya al entendimiento de la participación de los genes involucrados en la maduración de la guayaba; y pone a disposición secuencias parciales de ADNc que pueden utilizarse como marcadores o para estudios de expresión génica. Además, estas secuencias parciales pueden ser usadas como genes blanco a ser regulados para generar plantas cis-génicas que produzcan frutos con mayor vida de anaquel y mejores atributos de calidad, lo cual permitiría reducir el alto costo de su manejo en post-cosecha.

\section{CONCLUSIÓN}

Se identificaron cuatro ADNc relacionados con la maduración del fruto de guayaba de la variedad 'Media china'. Mediante análisis bioinformáticos, $P g P G 1$ presentó las principales características de las PGs y su mayor expresión ocurrió en el estadio maduro del fruto. Se caracterizaron dos genes de expansinas, $P g E X P 2$ y $P g E X P 3$, que contienen secuencias parciales de los dos dominios característicos de este tipo de enzimas. La expresión de PgEXP2 se observó desde el estadio verde 2 al de transición, mientras que $P g E X P 3$ se expresó en todos los estadios de maduración y en pedúnculo, con mayor nivel en el estadio maduro. Para PgACO1, la mayor expresión fue en el estadio de transición, con decremento en los estadios maduro y sobremaduro.

\section{AGRADECIMIENTOS}

A la Universidad Autónoma de Aguascalientes por el financiamiento del proyecto PIBT 10-3, y al Consejo Nacional de Ciencia y Tecnología (CONACYT) por la beca 
(228193). Al Dr. Saúl Padilla Ramírez del Instituto Nacional de Investigaciones Forestales, Agrícolas y Pecuarias (INIFAP) por su amable contribución con el material vegetal, y al Dr. Roberto Rico Martínez por su ayuda en la redacción de este artículo.

\section{BIBLIOGRAFÍA}

Agüiero G, E Rodríguez, L Díaz, N Bacallao, E Jiménez (2003) Aislamiento de un fragmento del ADNc ACC Oxidasa expresado durante la maduración del fruto de guayaba enana. Biotec. Veg. 3:187-189.

Agüero-Chapin G, H González-Díaz, R Molina, J Varona-Santos, E Uriarte, Y González-Díaz (2006) Novel 2D maps and coupling numbers for protein sequences. The first QSAR study of polygalacturonases; isolation and prediction of a novel sequence from Psidium guajava L. FEBS Lett. 580:723-730.

Altschul S F, W Gish, W Miller, E W Myers, D J Lipman (1990) Basic local alignment search tool. J. Mol. Biol. 215:403-410.

Artimo P, M Jonnalagedda, K Arnold, D Baratin, G Csardi, E de Castro, S Duvaud, V Flegel, A Fortier, E Gasteiger, A Grosdidier, C Hernandez, V Ioannidis, D Kuznetsov, R Liechti, S Moretti, K Mostaguir, N Redaschi, G Rossier, I Xenarios, H Stockinger (2012) ExPASy: SIB bioinformatics resource portal. $\mathrm{Nu}-$ cleic Acids Res. 40:W597-W603.

Asif M H, P Dhawan, P Nath (2000) A Simple procedure for the isolation of high quality RNA from ripening banana fruit. Plant Mol. Biol. Rep. 18:109-115.

Brummell D A, M H Harpster, P Dunsmuir (1999) Differential expression of expansin gene family members during growth and ripening of tomato fruit. Plant Mol. Biol. 39:161-169.

Choi D, H T Cho, Y Lee (2006) Expansins: expanding importance in plant growth and development. Physiol. Plant. 126:511-518.

Civello P M, A L T Powell, A Sabehat, A B Bennett (1999) An expansin gene expressed in ripening strawberry fruit. Plant Physiol. 121:1273-1279.

De la Cruz J, G Vela, L Dorantes, H S García (2010) Efecto del etileno sobre el ACC y el ACC oxidasa en la maduración de papaya 'Maradol'. Rev. Fitotec. Mex. 33:133-140.

Gorguet B, D Schipper, A van Lammeren, R G F Visser, A W van Heusden (2009) $p s-2$, the gene responsible for functional sterility in tomato, due to non-dehiscent anthers, is the result of a mutation in a novel polygalacturonase gene. Theor. Appl. Genet. 118:1199-1209.

Hadfield K A, A B Bennett (1998) Polygalacturonases: many genes in search of a function. Plant Physiol. 117:337-343.

Hadfield K A, J K C Rose, D S Yaver, R M Berka, A B Bennett (1998) Polygalacturonase gene expression in ripe melon fruit supports a role for polygalacturonase in ripening-associated pectin disassembly. Plant Physiol. 117:363-373.

Harrison E P, S J McQueen-Mason, K Manning (2001) Expression of six expansin genes in relation to extension activity in developing strawberry fruit. J. Exp. Bot. 52:1437-1446.
Hiwasa K, Y Kinugasa, S Amano, A Hashimoto, R Nakano, A Inaba, Y Kubo (2003) Ethylene is required for both the initiation and progression of softening in pear (Pyrus communis L.) fruit. J. Exp. Bot. 54:771-779.

Jain N, K Dhawan, S Malhotra, R Singh (2003) Biochemistry of fruit ripening of guava (Psidium guajava L.): compositional and en zymatic changes. Plant Foods Hum. Nutr. 58:309-315.

Larkin M A, G Blackshields, N P Brown, R Chenna, P A McGettigan, H McWilliam, F Valentin, I M Wallace, A Wilm, R Lopez, J D Thompson, T J Gibson, D J Higgins (2007) ClustalW and ClustalX version 2. Bioinformatics 23:2947-2948.

López-Gómez R, M A Gómez-Lim (1992) A method for extracting intact RNA from fruits rich in polysaccharides using ripe mango mesocarp. HortScience 27:440-442.

López-Gómez R, F Morales-Domínguez, O Mendoza A, M A GómezLim (2004) Identification of a genomic clone to ACC oxidase from papaya (Carica papaya L.) and expression studies. J. Agric. Food Chem. 52:794-800.

Momonoi K, K Shoji, K Yoshida (2007) Cloning and characterization of ACC oxidase genes from tulip. Plant Biotechnol. 24:241-246.

Nakatsuka A, S Murachi, H Okunishi, S Shiomi, R Nakano, Y Kubo, A Inaba (1998) Differential expression and internal feedback regulation of 1-aminocyclopropane-1-carboxylate synthase, 1-aminocyclopropane-1-carboxylate oxidase, and ethylene receptor genes in tomato fruit during development and ripening. Plant Physiol. 118:1295-1305.

Nishiyama K, M Guis, J K C Rose, Y Kubo, K A Bennett, L Wangjin, K Kato, K Ushijima, R Nakano, A Inaba, M Bouzayen, A Latche, J C Pech, A B Bennett (2007) Ethylene regulation of fruit softening and cell wall disassembly in Charentais melon. J. Exp. Bot. 58:1281-1290

Redondo-Nevado J, E Moyano, N Medina-Escobar, J L Caballero, J Muñoz-Blanco (2001) A fruit-specific and developmentally regulated endopolygalacturonase gene from strawberry (Fragaria $\times$ ananassa cv. Chandler). J. Exp. Bot. 52:1941-1945.

Rose J K, A B Bennett (1999) Cooperative disassembly of the cellulosexyloglucan network of plant cell wall: parallels between cell expansion and fruit ripening. Trends Plant Sci. 4:176-183.

Sambrook J, D W Russell (2001) Extraction, purification, and analysis of mRNA from eukaryotic cells: In: Molecular Cloning. A Laboratory Manual. J Argentine, N Irwin, K A Janssen, N Mclnerny, D Brown, S Schaefer (eds). 3d ed. Cold Spring Harbor Laboratory Press. New York. pp:7.46-7.50.

Sampedro J, D J Cosgrove (2005) The expansin superfamily. Genome Biol. 6:242.1-242.11.

Sharova E I (2007) Expansins: proteins involved in cell wall softening during plant growth and morphogenesis. Russ. J. Plant Physl. 54:713-727.

Tang X, H Wang, A S Brandt, W R Woodson (1993) Organization and structure of the 1-aminocyclopropane-1-carboxylate oxidase gene family from Petunia hybrida. Plant Mol. Biol. 23:1151 1164 .

Wu Y, R B Meeley, D J Cosgrove (2001) Analysis and expression of the $\alpha$-expansin and $\beta$-expansin gene families in maize. Plant Physiol. 126:222-232. 